\title{
Proposal to calculate the circadian component in lighting projects
}

\section{Propuesta para calcular la componente circadiana en los proyectos de iluminación}

\author{
A. Sánchez-Cano ${ }^{1,2, S^{*}}$, O.L. Pérez ${ }^{3}$, J. Aporta ${ }^{1,2}$ \\ 1. Applied Physics Department, University of Zaragoza, C/ Pedro Cerbuna, 12. 50009-Zaragoza, Spain \\ 2. Aragon Institute for Health Research (IIS Aragon), C/ San Juan Bosco, 13. 50009-Zaragoza, Spain \\ 3. WELL AP, Mount Sinai Hospital, New York, NY, USA \\ ${ }^{*}$ E-mail: anaisa@unizar.es \\ S: SEDOPTICA member
}

Received: 27/11/2018 Accepted: 27/02/2019

DOI: 10.7149/OPA.52.1.51015

\begin{abstract}
:
There are international norms which define methods for characterizing indoor luminaries in terms of photopic considerations and visual requirements, but lighting impacts in humans in others ways than those. Light in buildings is part of the environment, the discovery of the ipRGC cells in the human retina, and its associated action spectra, lead to the introduction of initial concepts such as circadian action functions and circadian action factors. An innovative methodology is proposed and evaluated to simulate potential melanopic contribution of the lit environment to the occupants of an indoor space. By performing measurements and efficiency simulations based on real LED luminaries, we are determining which parameters could be calculated in melanopic terms. Our measurements lead us to follow a method to differentiate and complement photopic and melanopic contributions of sources. These melanopic illuminance metrics require irradiance-wavelength-dependent measurements; recalculating polar curves, modifying data files, and spectral reflectance characterization to evaluate the melanopic contribution of the lit environment. We validate a simulation model through a comparison between the experimentally measured lighting values and those provided as output by commercial lighting design software DIALux.
\end{abstract}

Key words: ipRGC, melanopic illuminance, daylight equivalent melanopic illuminance, DIALux

\section{RESUMEN:}

Existen normas que definen los métodos para caracterizar fotométricamente las luminarias de interiores, pero la iluminación tiene un impacto en los seres humanos más allá de éstas. La luz en los edificios es parte del entorno, el descubrimiento de las células ipRGC en la retina humana y sus espectros de acción asociados, llevan a la introducción de conceptos tales como funciones de acción circadiana y factores de acción circadiana. En este trabajo, se propone y evalúa un método innovador para simular la posible contribución melanópica en espacios interiores. Al realizar mediciones y simulaciones de eficiencia basadas en unas luminarias LED reales, se han determinado qué parámetros podrían calcularse en términos melanópicos y se propone un método para diferenciar y complementar las contribuciones fotópicas y melanópicas de las fuentes de luz. Esta métrica de iluminancia melanópica requiere medidas de irradiancia espectral, recalcular las curvas polares, duplicar los archivos de datos y caracterizar la reflectancia espectral del entorno, para evaluar la contribución melanópica del espacio iluminado. Se valida un modelo de simulación a través de una comparación entre los valores de iluminación medidos experimentalmente y los proporcionados por el software de diseño de iluminación comercial DIALux.

Palabras clave: ipRGC, iluminancia melanópica, iluminancia melanópica equivalente luz día, DIALux 


\section{REFERENCES AND LINKS / REFERENCIAS Y ENLACES}

[1] G. Wyszecki, W. S. Stiles, Color science: concepts and methods, quantitative data and formulae, John Wiley \& Sons, New York etc. (2000).

[2] P. L. Kaufman, L. A. Levin, F. H. Adler, A. Alm, Adler's Physiology of the Eye, Elsevier Health Sciences (2011).

[3] China Academy of Building Research,"GB 50034-2013. Standard for Lighting Design of Buildings," (2013).

[4] CIBSE, "LG07/15 Lighting Guide 07: Offices-LG7," (2005).

[5] CEN/TC 169-Light and lighting, "EN 12464-1:2011. Lighting of work places - Part 1: Indoor work places," (2011).

[6] International WELL Building Institute (IWBI), "WELL Building Standard. LIGHT. Feature 53; Visual Lighting Design," (2017).

[7] American National Standards Institute and Illuminating Engineering Society of North America, (ANSI), "American National Standard Practice for Office Lighting. ANSI/ IES RP-1-12." (2012).

[8] M. Karlen, C. Spangler, J. R. Benya, Lighting design basics, John Wiley \& Sons (2017).

[9] D. Schreuder, Outdoor lighting: physics, vision and perception, Springer (2008).

[10] C. P. Wild, "Complementing the genome with an "exposome": the outstanding challenge of environmental exposure measurement in molecular epidemiology," Cancer Epidemiol Biomarkers Prev 14, 1847-1850 (2005).

[11] A. J. Lewy, T. A. Wehr, F. K. Goodwin, D. A. Newsome, S. P. Markey, "Light suppresses melatonin secretion in humans," Science 210,1267-1269 (1980).

[12] I. Provencio, M. D. Rollag, A. M. Castrucci, "Photoreceptive net in the mammalian retina. This mesh of cells may explain how some blind mice can still tell day from night," Nature 415, 493 (2002).

[13] G. C. Brainard, J. P. Hanifin, J. M. Greeson, B. Byrne, G. Glickman, E. Gerner, M. D. Rollag, "Action spectrum for melatonin regulation in humans: evidence for a novel circadian photoreceptor," J Neurosci 21, 6405-6412 (2001).

[14] K. Thapan, J. Arendt, D. J. Skene, "An action spectrum for melatonin suppression: evidence for a novel non-rod, non-cone photoreceptor system in humans," J Physiol 535, 261-267 (2001).

[15] D. Gall, "Circadiane Lichtgrößen und deren meßtechnische Erfassung," Licht 7, 860 (2002).

[16] M. S. Rea, M. G. Figueiro, A. Bierman, R. Hamner, "Modelling the spectral sensitivity of the human circadian system," Light Res Technol 44, 386-396 (2012).

[17] J. Enezi, V. Revell, T. Brown, J. Wynne, L. Schlangen, R. Lucas, "A "melanopic" spectral efficiency function predicts the sensitivity of melanopsin photoreceptors to polychromatic lights," J Biol Rhythms 26, 314323 (2011).

[18] R. J. Lucas, S. N. Peirson, D. M. Berson, T. M. Brown, H. M. Cooper, C. A. Czeisler, M. G. Figueiro, P. D. Gamlin, S. W. Lockley, J. B. O'Hagan, L. L. Price, I. Provencio, D. J. Skene, G. C. Brainard, "Measuring and using light in the melanopsin age," Trends Neurosci 37, 1-9 (2014).

[19] A. de Vries, J. L. Souman, B. de Ruyter, I. Heynderickx, Y. A. de Kort, "Lighting up the office: The effect of wall luminance on room appraisal, office workers' performance, and subjective alertness," Build Environ (2018).

[20] C. E. Ochoa, M. B. Aries, E. J. van Loenen, J. L. Hensen, "Considerations on design optimization criteria for windows providing low energy consumption and high visual comfort," Appl Energy 95, 238-245 (2012).

[21] C. E. Ochoa, M. B. C. Aries, J. L. M. Hensen, "State of the art in lighting simulation for building science: A literature review," J Build Perform Simul 5, 209-233 (2012).

[22] M. B. C. Aries, M. P. J. Aarts, and J. Van Hoof, "Daylight and health: A review of the evidence and consequences for the built environment," Light Res Technol 47, 6-27 (2015).

[23] Deutsches Institut für Normung, (DIN), "DIN SPEC 5031-100:2015-08. Optical radiation physics and illuminating engineering - Part 100: Melanopic effects of ocular light on human beings - Quantities, symbols and action spectra," (2015).

[24] European Committee for Standardization, (CEN), "NPR-CEN/TR 16791:2017. Quantifying irradiance for eye-mediated non-image-forming effects of light in humans," (2017). 
[25] W. Cai, J. Yue, Q. Dai, L. Hao, Y. Lin, W. Shi, Y. Huang, and M. Wei, "The impact of room surface reflectance on corneal illuminance and rule-of-thumb equations for circadian lighting design," Build Environ (2018).

[26] G. Esquiva, P. Lax, J. J. Perez-Santonja, J. M. Garcia-Fernandez, N. Cuenca, "Loss of MelanopsinExpressing Ganglion Cell Subtypes and Dendritic Degeneration in the Aging Human Retina," Front Aging Neurosci 9, 79 (2017).

[27] L. Bellia, A. Pedace, G. Barbato, "Lighting in educational environments: An example of a complete analysis of the effects of daylight and electric light on occupants," Build Environ 68, 50-65 (2013).

[28] V. Mottram, B. Middleton, P. Williams, J. Arendt, "The impact of bright artificial white and 'blueenriched' light on sleep and circadian phase during the polar winter," J Sleep Res 20, 154-161 (2011).

[29] J. van Hoof, M. P. J. Aarts, C. G. Rense, A. M. C. Schoutens, "Ambient bright light in dementia: Effects on behaviour and circadian rhythmicity," Build Environ 44, 146-155 (2009).

[30] M. S. Rea, M. G. Figueiro, J. D. Bullough, "Circadian photobiology: An emerging framework for lighting practice and research," Light Res Technol 34, 177-187 (2002).

[31] Y. Kim, A. Choi, J. Jeong, "Applying micro genetic algorithm to numerical model for luminous intensity distribution of planar prism LED luminaire," Opt Commun 293, 22-30 (2013).

[32] D. Kim, B. Han, Y. Kim, "Degradation analysis of secondary lens system and its effect on performance of LED-based luminaire," Microelectron Reliab 54, 131-137 (2014).

[33] P. Khademagha, M. Aries, A. Rosemann, E. Van Loenen, "Why directionality is an important light factor for human health to consider in lighting design?" International Journal of Sustainable Lighting 18, 3-8 (2016).

[34] R. J. Reiter, "Pineal melatonin: Cell biology of its synthesis and of its physiological interactions," Endocr Rev 12,151-180 (1991).

[35] C. A. Czeisler, J. F. Duffy, T. L. Shanahan, E. N. Brown, J. F. Mitchell, D. W. Rimmer, J. M. Ronda, E. J. Silva, J. S. Allan, J. S. Emens, D. Dijk, R. E. Kronauer, "Stability, precision, and near-24-hour period of the human circadian pacemaker," Science 284, 2177-2181 (1999).

[36] S. W. Lockley, E. E. Evans, F. A. J. L. Scheer, G. C. Brainard, C. A. Czeisler, D. Aeschbach, "Short-wavelength sensitivity for the direct effects of light on alertness, vigilance, and the waking electroencephalogram in humans," Sleep 29, 161-168 (2006).

\section{Introducción}

Llamamos luz a la radiación electromagnética comprendida entre los $380 \mathrm{~nm}$ y los $780 \mathrm{~nm}$, intervalo en el que son sensibles los fotorreceptores existentes en la retina del ojo humano y que son los responsables de la detección de la luz reflejada o transmitida (de forma regular o difusa) por los diferentes objetos [1]. Esas células fotorreceptoras, conos y bastones, captan la información, tanto de luminancia como de color, contenida en cada punto de la imagen, al igual que lo hace el sensor de la cámara fotográfica. Dicha información se transmite, de forma más o menos compleja, al cerebro, donde se procesa y almacena para que el ser humano pueda interpretarla y le sea útil para realizar las diferentes funciones que en cada momento precisa [2]. Esta sería la llamada vía visual o formadora de imagen, que es la más conocida y a través de la cual se estima que los seres humanos recibimos hoy más del $85 \%$ de la información que procesan nuestros sentidos. Los múltiples estudios de esta ruta óptica han permitido establecer de forma correcta todos los mecanismos relacionados con la visión, así como las necesidades y calidades de la iluminación generadas para cada actividad y que aparecen en la normativa vigente [3-7]. Esa vía, por tanto, es la única que ha estado presente, desde que la luz artificial ha entrado a formar parte de nuestra vida diaria, en el desarrollo de toda la tecnología del sector de la iluminación y en el cuerpo normativo de la luminotecnia, con el que se han venido elaborando y valorando los proyectos de iluminación, tanto de interiores como de exteriores [8,9]. La luz que recibe el ojo, sin embargo, interviene no sólo en esa formación visual, sino que forma parte del denominado exposoma [10]. Es imprescindible, en concreto, en la sincronización del sistema circadiano, sistema encargado de poner cada día en hora nuestro reloj biológico, en concordancia con los ciclos del día y la noche (luz-oscuridad) [11]. Si bien los efectos no visuales de la luz, también llamados no formadores de imágenes (NIF) ya se conocían y reivindicaban en el pasado, sólo ha sido posible tener una comprensión inicial de las condiciones de iluminación apropiadas (tiempo, dosis, espectros, direccionalidad, ...) desde el reciente descubrimiento de la melanopsina, los espectros de acción 
de la melanopsina y las células humanas ipRGC (intrinsically photosensitive retinal ganglion cells) que median en esta respuesta no visual. La melanopsina en humanos fue descubierta por Provencio et al. [12], y su espectro de acción fue propuesto por primera vez simultáneamente por Brainard et al., (máximo a 460 $\mathrm{nm}$ ), y Thapan et al., (máximo a $456 \mathrm{~nm}$ ), en 2001 [13,14]. Posteriormente se han propuesto diferentes espectros de acción y métricas para estimar la potencial contribución melanópica de la iluminación. Una de las primeras aproximaciones fue realizada por Gall [15], con un espectro concreto de supresión de la melatonina, e introduciendo los términos de "espectro de acción circadiana" y "factor de acción circadiana". A continuación, Rea et al. [16], construyó un modelo matemático diseñado para ser consistente con los datos obtenidos de la psicofísica, la electrofisiología y la neuroanatomía, con nuevos parámetros como los estímulos circadianos (CS) y la luz circadiana (CLA). Análogamente Enezi et al. [17] realizaron experimentos con ratones para determinar empíricamente cómo ponderar la potencia óptica en cada longitud de onda de acuerdo con un nomograma a $480 \mathrm{~nm}$, y al integrar en todo el espectro, podían predecir la sensibilidad de la melanopsina en una variedad de estímulos policromáticos. Como Lucas et al. [18], cuyos métodos de medición de luz cuantifican la irradiancia efectiva para cada una de las longitudes de onda que llegan al sistema visual para integrarlas y valorar la repercusión que tienen en conjunto.

En base a la influencia de la luz en la cronobiología humana, el control de la cantidad de luz recibida por el ojo, procedente de las diferentes luminarias y superficies presentes en los espacios de interior, es de capital importancia [19]. La luz artificial es casi la única que recibe el ojo a lo largo de muchas horas, sobre todo durante el invierno y en países de latitudes altas. A ello ha contribuido también el abaratamiento de la luz eléctrica y la popularización del aire acondicionado a partir de los 50 que ha hecho que muchos arquitectos comenzaran a diseñar una parte significativa de edificios para funcionar casi exclusivamente con luz artificial y con criterios de eficiencia energética [20,21]. Esto, unido a las tendencias que en cada momento primaban sobre las envolventes de los edificios, sus propias escalas y morfometrías, hacían difícil otro tipo de planteamiento para un correcto control de la iluminación a lo largo de toda la jornada laboral [22].

Todas las métricas descritas hasta el momento se basan principalmente en los espectros de acción de los fotorreceptores. La contribución de este trabajo es proporcionar una metodología fácil de aplicar que permita a los investigadores, arquitectos o diseñadores de iluminación simular la posible contribución melanópica en ambientes interiores iluminados, de acuerdo con dichas métricas. Una de las principales ventajas de esta propuesta es que la metodología es métrica-independiente, pero teniendo en cuenta que este documento no va a discutir la validez científica de las diferentes métricas propuestas. El método que se propone es utilizar software disponible comercialmente, en este caso DIALux (DIAL, Lüdenscheid, Germany), para la planificación, el cálculo y la simulación de la iluminación interior. Este software utiliza archivos eulumdat.ldt, estándar oficial en Europa para el intercambio de fotometría, que almacenan entre otros datos intensidades luminosas relativas, Temperatura de Color Correlacionada o Índice de Reproducción Cromática. En base a estos nuevos archivos eulumdat.ldt, tanto fotópicos como melanópicos, el objetivo final sería poder optimizar las simulaciones de iluminación en cualquier estancia. Este proceso requiere la inclusión del espectro melanópico, además del fotópico, en los archivos de datos de las fuentes de luz y los valores de reflectancia de los cálculos fotométricos clásicos.

\section{Material y métodos}

El primer paso sería caracterizar la luminaria; los datos radiométricos espectrales se pueden obtener de los fabricantes o a través de laboratorios acreditados donde se disponga de gonioespectrómetro. En este trabajo, se realizaron mediciones radiométricas y fotométricas en el Departamento de Física Aplicada de la Universidad de Zaragoza y en el Laboratorio Grupolux en Zaragoza, España. Se dispuso una luminaria con cuarenta y cinco LEDs blancos de $6500 \mathrm{~K}$ y otros cuarenta y cinco LED blancos de $2700 \mathrm{~K}$ que funcionaban en cuatro configuraciones diferentes. Ambos tipos de LED tenían unas dimensiones $5.6 \times 3.0 \times 0.9 \mathrm{~mm}(\mathrm{~L} \times$ $\mathrm{W} \times \mathrm{H}$ ), el material del chip era InGaN, su ángulo de visión de $120^{\circ}$, y la luminaria $(300 \times 300 \mathrm{~mm})$ se podía instalar con o sin difusor (Tabla 1).

Las curvas fotométricas se midieron con un sistema fotogoniométrico de fabricación propia, Figura 1. Se instaló la luminaria y se posicionó el detector rotatorio calibrado $\mathrm{V}(\lambda)$ (cabeza del fotómetro número de serie TH15-C10-x0-D8, PRC-Krochmann, Berlín, Alemania con trazabilidad certificada por el Instituto Física Aplicada-CSIC) situado a $2.5 \mathrm{~m}$ del plano fotométrico de la luminaria. Se realizaron las mediciones en torno a tres ejes: $0^{\circ}, 45^{\circ}$ y $90^{\circ}$ (C0-C180, C45-C225 y C90-C270). 
TABLA 1. Configuraciones de las luminarias ensayadas

\begin{tabular}{|c|c|c|c|c|}
\hline & Modo I (C.C.) & $\mathrm{CCT}(\mathrm{K})$ & $\mathrm{CRI}\left(\mathrm{R}_{\mathrm{a}}\right)$ & $\mathrm{R}_{9}$ \\
\hline $50-50$ sin difusor & $\begin{array}{c}\text { Cool } 350 \mathrm{~mA} \\
\text { Warm } 350 \mathrm{~mA}\end{array}$ & 4257 & 86 & 31 \\
\hline $50-50$ con difusor & Cool 350mA & 4077 & 86 & 32 \\
\hline $50-50$ con alrusor & Warm $350 \mathrm{~mA}$ & 4115 & 85 & 27 \\
\hline 100 Cool con difusor & Cool 700mA & 6808 & 84 & 19 \\
\hline 100 CoOr cont untus or & Warm Off & 6770 & 85 & 23 \\
\hline 100 Warm con difusor & $\begin{array}{c}\text { Cool Off } \\
\text { Warm 700mA } \\
\end{array}$ & 2735 & 80 & 5 \\
\hline
\end{tabular}

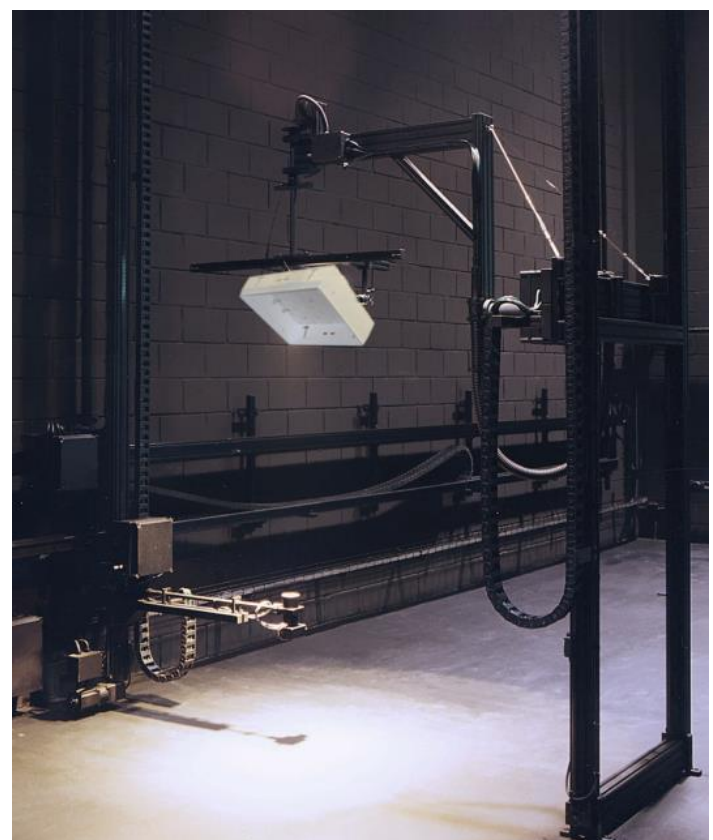

Fig.1. Sistema fotogoniométrico usado para caracterizar la luminaria en sus cuatro modos.

Para validar la contribución circadiana, es necesario utilizar una función melanópica como función de ponderación para generar archivos fotométricos a partir de las fuentes de iluminación que se elijan en cualquier diseño de iluminación. En fotometría, la luminosidad fotópica CIE $1931 \mathrm{~V}(\lambda)$ [1] es obligatoria para los cálculos fotópicos y, en este trabajo, para calcular las contribuciones melanópicas se ha elegido la función $S(\lambda)$ descrita por Lucas et al. [18]. Sin embargo, otras funciones de luminosidad melanópica se deberían analizar para calcular iluminancias tanto fotópicas como melanópicas, E (lux) y Emel (lux-mel), respectivamente, en un plano específico y comparar todos los parámetros descritos en este trabajo. Con esta premisa como punto de partida, para comparar las distribuciones espaciales fotópicas y melanópicas, se midieron y calcularon las diferentes curvas polares de las luminarias.

La distribución espectral de potencia (SPD) de estas luminarias se midió, dependiendo del ángulo de rotación, con un espectrorradiómetro calibrado (modelo Avaspec-1024, Avantes, Apeldoorn, Países Bajos, con calibración NPL E01110063/DDK y trazabilidad NIST). Este equipo se utilizó para medir la irradiancia espectral $(\mu \mathrm{W} / \mathrm{cm} 2)$ de $380 \mathrm{~nm}$ a $780 \mathrm{~nm}$, a $2.5 \mathrm{~m}$ del plano fotométrico, y para calcular los parámetros fotométricos. La relación entre la irradiancia y la posición angular se midió en pasos de $10^{\circ}$.

Adicionalmente, se tuvieron en cuenta los valores de reflectancia melanópica para los diferentes elementos de un espacio real donde se instalaron las luminarias. Los techos, las paredes, los suelos y la reflectancia de los muebles se deben evaluar siguiendo el mismo concepto que las fuentes de luz. Las mediciones de reflectancia en función de la longitud de onda se realizaron con un espectrofotómetro CM-700d (Konica Minolta Inc, Tokio, Japón) y la reflectancia ( $\rho)$ se calculó con (1). 


$$
\begin{gathered}
\begin{array}{c}
\text { ÓPTICA PURA Y APLICADA } \\
\text { www.sedoptica.es }
\end{array} \\
\rho=\frac{\int_{\lambda} \rho(\lambda) \operatorname{SPD}(\lambda) D(\lambda) d \lambda}{\int_{\lambda} \operatorname{SPD}(\lambda) D(\lambda) d \lambda}
\end{gathered}
$$

Donde $\rho(\lambda)$ es la reflectancia, $\operatorname{SPD}(\lambda)$ es la distribución espectral del iluminante, y $\mathrm{D}(\lambda)$ es la curva de respuesta espectral (fotópica, $\mathrm{V}(\lambda)$ o melanópica, $S(\lambda)$ ). La integración se llevó a cabo en toda la región de espectro en la que se definieron ambas funciones de respuesta espectral. La reflectancia fotópica $(\rho)$ y la reflectancia melanópica $\left(\rho_{\mathrm{mel}}\right)$ se calcularon en ambientes blanco, gris, azul y rojo. La Fig. 2 muestra la reflectancia espectral de cada pared utilizada en los experimentos.

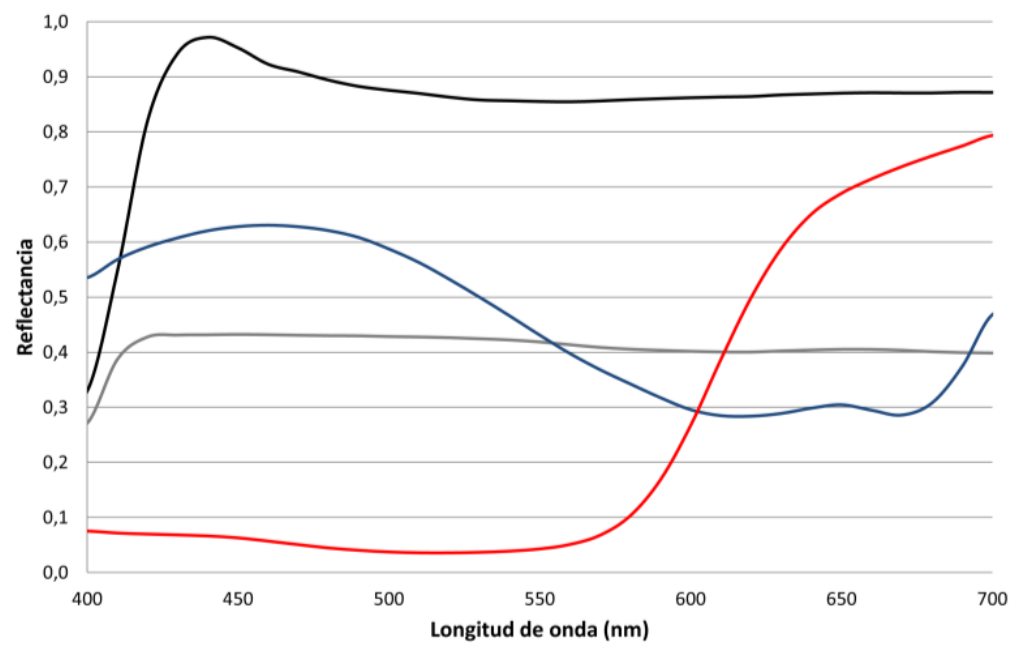

Fig.2. Reflectancia espectral de diferentes espacios. Paredes blancas (negro), paredes grises (gris), paredes rojas (rojo) y paredes azules (azul)

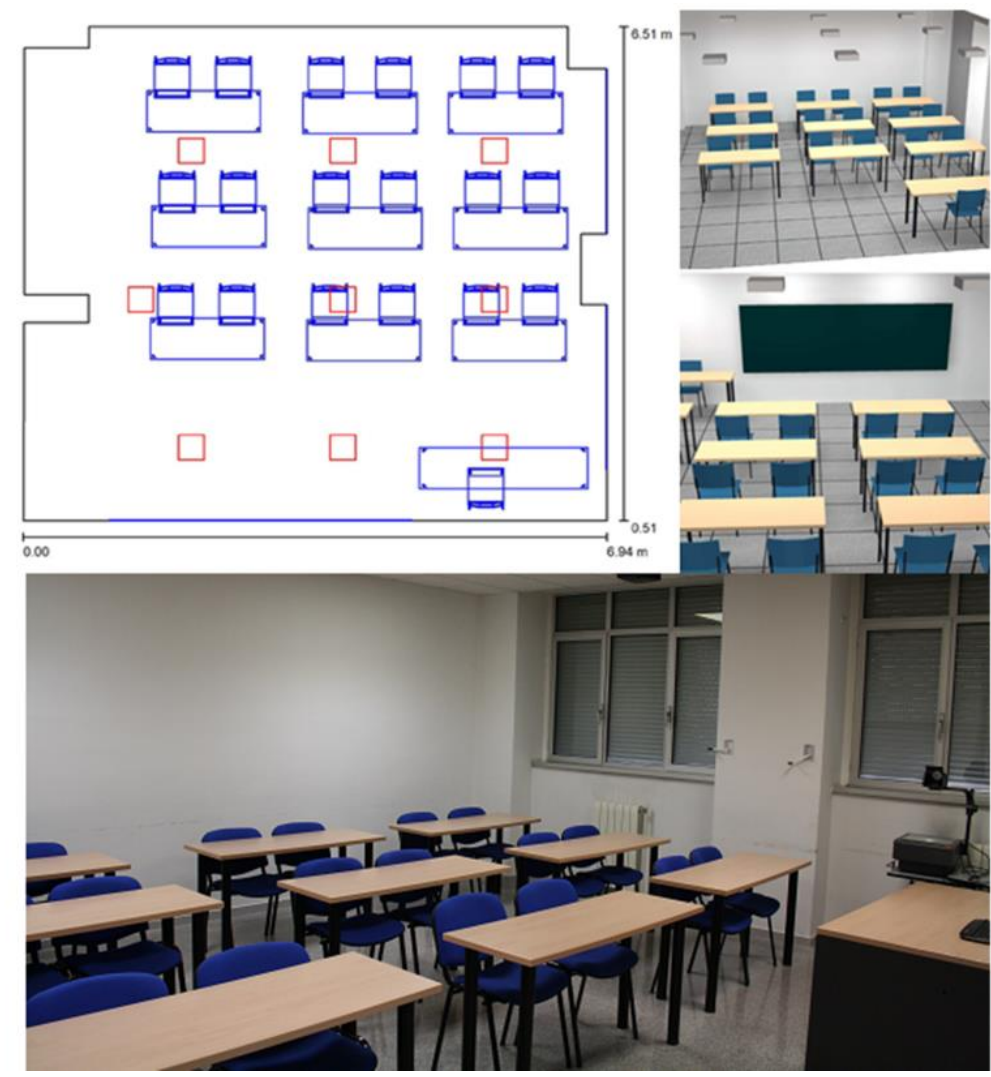

Fig.3. Arriba: Simulación en DIALux de un espacio docente. Izquierda: en rojo las nueve luminarias y en azul el mobiliario es colar. Derecha: vistas trasera y frontal del rendering de la simulación. Abajo: Imagen real del aula simulada. 
En la base a todos esos parámetros descritos, se realizó un proyecto de iluminación con el software comercial DIALux. Con este programa se simuló un entorno académico realista, con nueve luminarias de idénticas características a las que se habían ensayado con anterioridad, y se ubicaron en el techo de una sala virtual (Fig.3). La sistematización de la componente melanópica en este tipo de software requiere el cálculo de la pseudoIntensidad melanópica, usando la curva de respuesta espectral melanópica $S(\lambda)$ en lugar de la fotópica $V(\lambda)$, y teniendo en cuenta el factor de normalización de 1.218 por la diferencia de área entre ambas curvas. De esta manera se crearon dos tipos de archivos eulumdat.ldt, uno con intensidades fotópicas y otro con melanópicas, y se realizó el análisis con ambos.

Para cada configuración, se evaluaron y registraron los niveles de iluminancia para comparar con las mediciones experimentales realizadas en un aula existente con las mismas características que la sala replicada. Se instalaron nueve luminarias con un controlador DALI (Digital Addressable Lighting Interface) en un aula con las mismas dimensiones y muebles que la escena virtual. Las mediciones de iluminancia se tomaron en puntos estratégicos para compararlos con los simulados.

\section{Resultados}

\subsection{Medidas fotogoniométricas y comparación entre estándares.}

Se eligieron cuatro configuraciones distintas para realizar los ensayos de una misma luminaria como se refleja en la Tabla2.

TABLA 2. Características de los cuatro modos de la luminaria ensayada

\begin{tabular}{|c|c|cc|}
\hline \hline & Modo I (C.C.) & Flujo fotópico (lm) & Flujo melanópico (lm-mel) \\
\hline \hline 50-50 sin difusor & $\begin{array}{c}\text { Cool 350mA } \\
\text { Warm 350mA }\end{array}$ & 2014 & 1430 \\
50-50 con difusor & $\begin{array}{c}\text { Cool 350mA } \\
\text { Warm 350mA } \\
\text { Cool 700mA } \\
\text { Warm Off }\end{array}$ & 1692 & 1211 \\
100 Warm con difusor & $\begin{array}{c}\text { Cool Off } \\
\text { Warm 700mA }\end{array}$ & 1567 & 549 \\
\hline \hline
\end{tabular}

Experimentalmente, las curvas polares se midieron con un goniofotómetro a $2.5 \mathrm{~m}$ del plano fotométrico. La Fig.4 muestra los resultados en dos configuraciones diferentes, 50-50 sin difusor y 50-50 con difusor, para contribuciones tanto fotópicas como melanópicas. La primera configuración, 50-50 sin difusor alcanza $\mathrm{I}_{\max }=850 \mathrm{~cd}$ y $624 \mathrm{~cd}-\mathrm{mel}$, mientras que la misma luminaria con difusor alcanza $\mathrm{I}_{\max }=646 \mathrm{~cd}$ y $459 \mathrm{~cd}-\mathrm{mel}$.

A partir del SPD medido en plano C90-C270 de cada modo de la luminaria, se realizaron cálculos teóricos de los parámetros melanópicos involucrados en el procesamiento no visual de acuerdo con algunos estándares actuales, WELL [6], DIN [23], CEN [24] y con los métodos anteriormente descritos por Rea et al. [16] y Lucas et al. [18]. El resumen de la Tabla 3 valida que los resultados no se pueden comparar fácilmente cuando se establece una iluminancia fotópica fija con una luminaria real, siendo necesario un estudio más amplio.

El estudio de la dependencia del SPD con el ángulo de rotación se realizó en el plano C90-C270 dado que en los planos C0 y C45 se encontró un comportamiento similar. La Fig.5 muestra la irradiancia espectral absoluta, de $380 \mathrm{~nm}$ a $780 \mathrm{~nm}$, para cada una de las configuraciones. Las medidas se tomaron de $0^{\circ}$ a $80^{\circ}$, en pasos de $10^{\circ}$. Se puede observar que la forma de la pendiente se conserva en cada paso, por lo que las relaciones CCT, CRI, R9 y E El/E permanecen también constantes; esto demuestra, para cada luminaria, la independencia de dichos parámetros con el ángulo. 


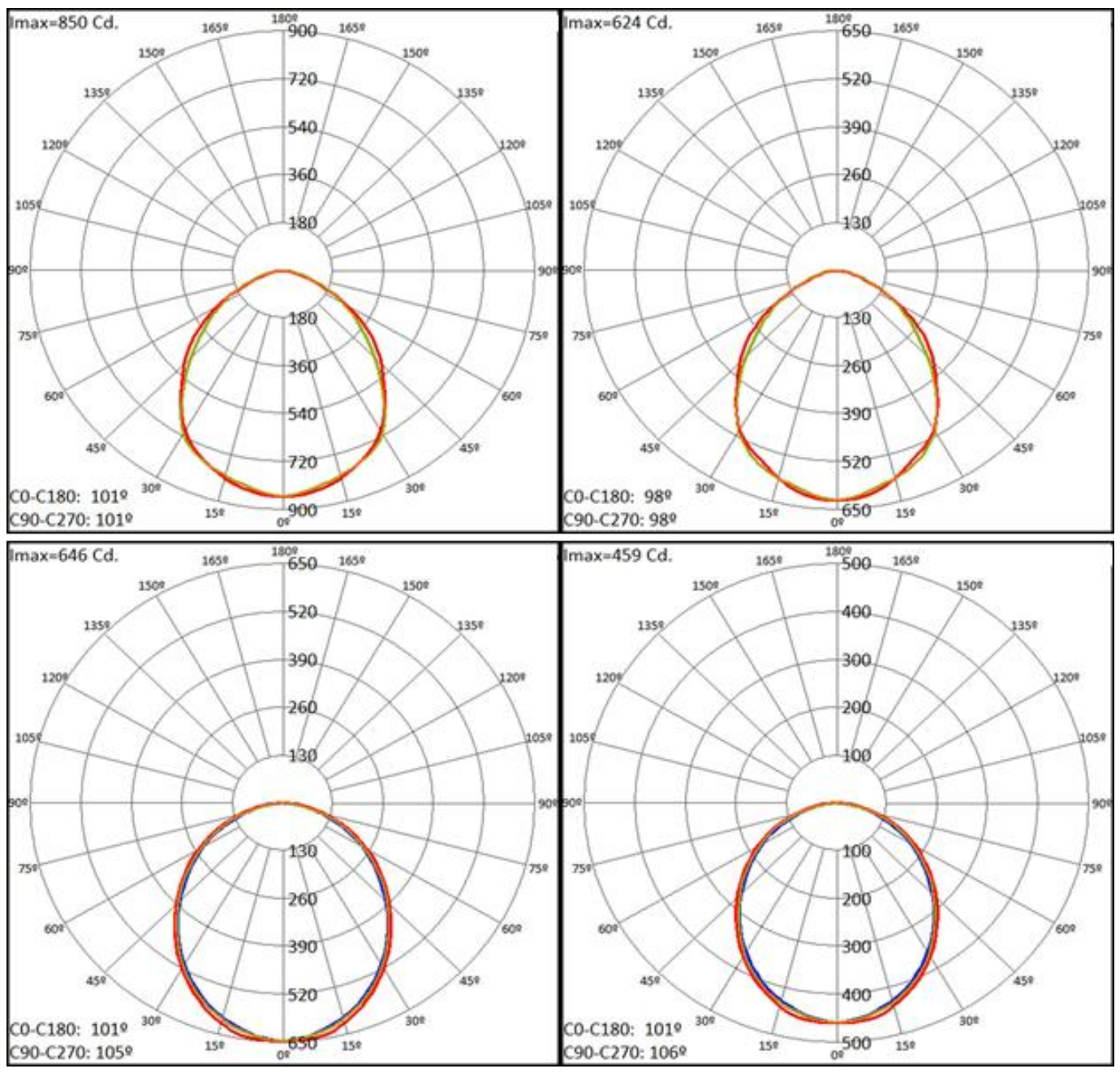

Fig.4. Curvas polares. Arriba 50-50 sin difusor (fotópico/melanópico) y abajo 50-50 con difusor.

TABLA 3. Contribución fotópica y melanópica para diferentes métricas y comparación entre estándares. $\mathbf{k}_{\text {mel,trans }}$ es el factor de corrección de la transmisión de los medios oculares, corregido por edad (observador de referencia 32 años)

\begin{tabular}{|c|c|c|c|c|c|c|c|c|c|c|}
\hline & \multicolumn{3}{|c|}{ DIN SPEC 5031-100:2005 [23] } & \multicolumn{7}{|c|}{ NPR-CEN/TR 16791:2017 [24] } \\
\hline & $\begin{array}{c}E \\
\text { (lux) }\end{array}$ & $\begin{array}{c}\text { Equivalente } \\
\text { melanópico- } \\
\text { luz día, } E_{\text {mel }} \\
\text { (lux-mel) }\end{array}$ & $\begin{array}{c}\text { Factor de } \\
\text { eficiencia } \\
\text { equivalente } \\
\text { melanópico- } \\
\text { luz día }\end{array}$ & $\begin{array}{c}k_{\text {mel,trans }} \\
(20 \\
\text { años })\end{array}$ & $\begin{array}{c}k_{\text {mel,trans }} \\
(32 \\
\text { años })\end{array}$ & $\begin{array}{c}\mathrm{k}_{\text {mel,trans }} \\
\text { (65 } \\
\text { años) }\end{array}$ & $\begin{array}{c}E \\
\text { (lux) }\end{array}$ & $\begin{array}{c}\mathrm{E}_{\text {mel }}(20) \\
\text { (lux- } \\
\text { mel) }\end{array}$ & $\begin{array}{c}\mathrm{E}_{\text {mel }}(32) \\
\text { (lux- } \\
\text { mel) }\end{array}$ & $\begin{array}{c}\mathrm{E}_{\text {mel }}(65) \\
\text { (lux- } \\
\text { mel) }\end{array}$ \\
\hline $50-50$ sin difusor & 129 & 85.75 & 0.665 & 1.079 & 1.000 & 0.696 & 129 & 92.56 & 85.79 & 59.71 \\
\hline $50-50$ con difusor & 99 & 63.37 & 0.640 & 1.077 & 1.000 & 0.701 & 99 & 68.24 & 63.36 & 44.42 \\
\hline 100 Cool con difusor & 93 & 83.25 & 0.895 & 1.083 & 1.000 & 0.682 & 93 & 90.14 & 83.24 & 56.77 \\
\hline 100 Warm con difusor & 87 & 32.16 & 0.370 & 1.063 & 1.000 & 0.747 & 87 & 34.22 & 32.19 & 24.05 \\
\hline
\end{tabular}

\begin{tabular}{|c|c|c|c|c|c|c|c|c|c|}
\hline & \multicolumn{3}{|c|}{$\begin{array}{l}\text { WELL Building Standard v1.0 } \\
\text { (rev. January 2017) [6] }\end{array}$} & \multicolumn{3}{|c|}{ Lucas et al. [18] } & \multicolumn{3}{|c|}{ Rea et al. [16] } \\
\hline & $\begin{array}{l}E \\
(\operatorname{lux})\end{array}$ & $\begin{array}{c}\mathrm{E}_{\mathrm{mel}} \\
\text { (lux-mel) }\end{array}$ & $\begin{array}{c}E_{\text {mel }} / E \\
\text { Ratio }\end{array}$ & $\begin{array}{c}E \\
(\operatorname{lux})\end{array}$ & $\begin{array}{l}\mathrm{E}_{\mathrm{mel}} \\
\text { (lux- } \\
\text { mel) }\end{array}$ & $\begin{array}{c}\mathrm{E}_{\mathrm{mel}} / \mathrm{E} \\
\text { Ratio }\end{array}$ & $\begin{array}{l}\mathrm{CL} \\
(\operatorname{lux})\end{array}$ & CLA & CS \\
\hline $50-50$ sin difusor & 129 & 95 & 0.74 & 128.43 & 94.27 & 0.734 & 129 & 106.18 & 0.146 \\
\hline $50-50$ con difusor & 99 & 70 & 0.71 & 96.30 & 68.06 & 0.707 & 99 & 72.28 & 0.103 \\
\hline 100 Cool con difusor & 93 & 92 & 0.99 & 93.19 & 92.10 & 0.988 & 93 & 132.71 & 0.177 \\
\hline 100 Warm con difusor & 87 & 37 & 0.42 & 82.18 & 33.54 & 0.408 & 82 & 60.79 & 0.087 \\
\hline
\end{tabular}



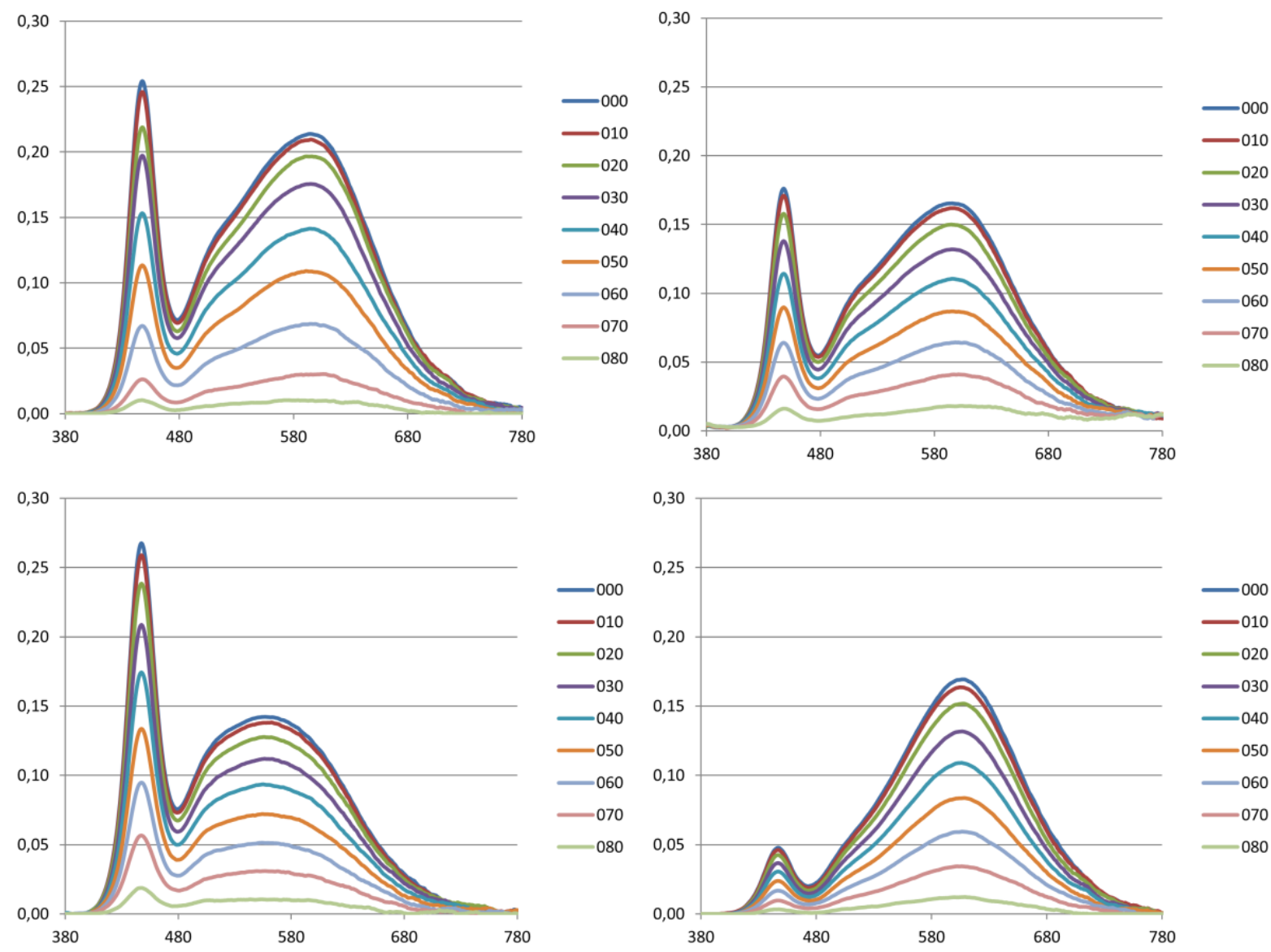

Fig.5. Dependencia del SPD con el ángulo de medida en el plano C090. Eje vertical, Irradiancia $(\mu \mathrm{W} / \mathrm{cm} 2)$; eje horizontal, longitud de onda (nm). Arriba-izquierda, 50-50 sin difusor; arriba-derecha, 50-50 con difusor; abajo-izquierda, 100 Cool con difusor; abajoderecha, 100 Warm con difusor.

\subsection{Cálculos y simulación con DIALux.}

Como caso de estudio, se generó con DIALux un aula docente de $6.94 \times 6.51 \mathrm{~m}$ con muebles, paredes y techos blancos, y suelo gris, Fig.3. Se colocaron en el techo nueve luminarias idénticas en el modo 50-50 con difusor, a una altura de $2.90 \mathrm{~m}$ sobre el suelo. La reflectancia fotométrica total del suelo, las paredes y el techo se midió experimentalmente con un colorímetro y se introdujo en los parámetros del software. Los cálculos en este programa a $120 \mathrm{~cm}$ desde el suelo (plano vertical, $E_{V}$ ) y a $70 \mathrm{~cm}$ desde el suelo (plano horizontal, $E_{H}$ ) se realizaron basándose en la cuadrícula de cálculo más precisa y en la luz directa y las interreflexiones sucesivas de las luminarias. No se consideró la luz del día en esta simulación, sólo la iluminación artificial, y para simplificar los cálculos, se consideró exclusivamente la función melanópica descrita por Lucas et al. [18], aunque estos cálculos podrían realizarse con cualquier otra métrica. Para verificar las simulaciones, se tomaron valores experimentales de irradiancia con un espectrorradiómetro y se calcularon las iluminancias fotópicas y melanópicas en los mismos puntos que en DIALux. La Tabla 4 muestra las iluminancias, obtenidas experimentalmente y después de la representación del espacio, la relación $\mathrm{E}_{\mathrm{mel}} / \mathrm{E}$ y las relaciones $\mathrm{E}_{\mathrm{V}} / \mathrm{E}_{\mathrm{H}}$.

TABLA 4. Valores de iluminancia medida de forma experimental y simulada con DIALux.

\begin{tabular}{|c|c|cc|c|c|c|}
\hline \hline & \multicolumn{3}{|c|}{ Simulación con DIALux } \\
\hline \hline & $E$ (lux) & $E_{\text {mel }}$ (lux-mel) & $E_{\text {mel }} /$ E Ratio & E (lux) & $E_{\text {mel }}$ (lux-mel) & $E_{\text {mel }} / E$ Ratio \\
$E_{H}(70 \mathrm{~cm})$ & 282 & 192 & 0.68 & 226 & 161 & 0.72 \\
$E_{V}(120 \mathrm{~cm})$ & 125 & 83 & 0.66 & 111 & 78 & 0.71 \\
$E_{v} / E_{H}$ ratio & 0.44 & 0.43 & & 0.49 & 0.49 & \\
\hline \hline
\end{tabular}


Para evaluar y comparar la relación entre la iluminancia fotópica en un plano horizontal y la cantidad de iluminancia vertical que puede alcanzar el plano corneal, se puede definir y calcular el parámetro $\left(\mathrm{E}_{\mathrm{Vmel}} / \mathrm{E}_{\mathrm{H}}\right)$. En nuestras mediciones simuladas fue 78/226 $=0.35$, y en las experimentales 83/282 $=0.29$.

La reflectancia fotópica total, $\rho$, de las paredes, techo y suelo en un espacio concreto también podría diferenciarse de su reflectancia equivalente melanópica, este nuevo parámetro se puede calcular con la expresión estándar sustituyendo $V(\lambda)$ por $S(\lambda)$. Como se ha descrito anteriormente, se caracterizaron espectrofotométricamente cuatro tipos de paredes; blancas, grises, azules y rojas. La $\rho$ calculada en cada caso fue $\rho_{\text {blanco }} 0.862, \rho_{\text {gris }} 0.413$, $\rho_{\text {azul }} 0.400$ y $\rho_{\text {rojo }} 0.145$, y la reflectancia melanópica fue, respectivamente, $\rho_{\text {mel-blanco }} 0.895, \rho_{\text {mel-gris }} 0.428, \rho_{\text {mel-azul }} 0.571$ y $\rho_{\text {mel-rojo }} 0.045$.

Se realizaron simulaciones con DIALux, modificando los archivos, incluidos los SPD, las reflectancias espectrales $\left(\rho\right.$ o $\left.\rho_{\text {mel }}\right)$ y $V(\lambda)$ o $S(\lambda)$ según fuesen cálculos fotópicos o melanópicos. Además, en el aula se midieron las iluminancias horizontales para comparar con los valores obtenidos con DIALux. Los resultados en iluminancia fotópica, medidos experimentalmente y simulados, fueron mayores con paredes blancas, seguidos con valores similares con paredes azules y grises, y se encontraron valores más bajos con superficies rojas (Experimental: $\mathrm{E}_{\text {blanco }} 239.3$ lux, $\mathrm{E}_{\text {azul }} 209.0$ lux y $\mathrm{E}_{\text {gris }} 205.2$ lux, $\mathrm{E}_{\text {rojo }} 191.8$ lux; simulado: Eblanco 231.8 lux, Eazul 190.0 lux y Egris 190.0 lux, $E_{\text {rojo }} 173.6$ lux).

También se puede evaluar la iluminancia melanópica y se pueden encontrar diferencias en el comportamiento de las paredes de color, se alcanzan valores más altos con paredes blancas, seguidos de las áreas circundantes de azul, gris y rojo. (Experimental: $\mathrm{E}_{\text {mel-blanco }} 169.5$ lux-mel, $\mathrm{E}_{\text {mel-azul }} 156.0$ lux-mel y $\mathrm{E}_{\mathrm{mel}}$ gris 146.6 lux-mel, $E_{\text {mel-rojo }} 132.2$ lux-mel; simulado: $E_{\text {mel-blanco }} 169.2$ lux-mel, $E_{\text {mel-azul }} 145.3$ lux-mel, $E_{\text {mel-gris }}$ 137.3 lux-mel, $\mathrm{E}_{\text {mel-rojo }} 120.4$ lux-mel).

Comparando el rendimiento melanópico de cada situación con respecto a la iluminancia fotópica, el ambiente azul tuvo una relación más alta (relación $\mathrm{E}_{\mathrm{mel}} / \mathrm{E}>0.75$ ), el blanco y el gris fueron equivalentes (relación $\mathrm{E}_{\mathrm{mel}} / \mathrm{E}$ de 0.71 a 0.73 ) y el valor más bajo se encontró con paredes rojas (relación $\mathrm{E}_{\mathrm{mel}} / \mathrm{E}<0.70$ ).

En la Fig. 6 se explica a modo de resumen, la propuesta realizada para incluir los cálculos melanópicos, además de los fotópicos, en los proyectos de iluminación.

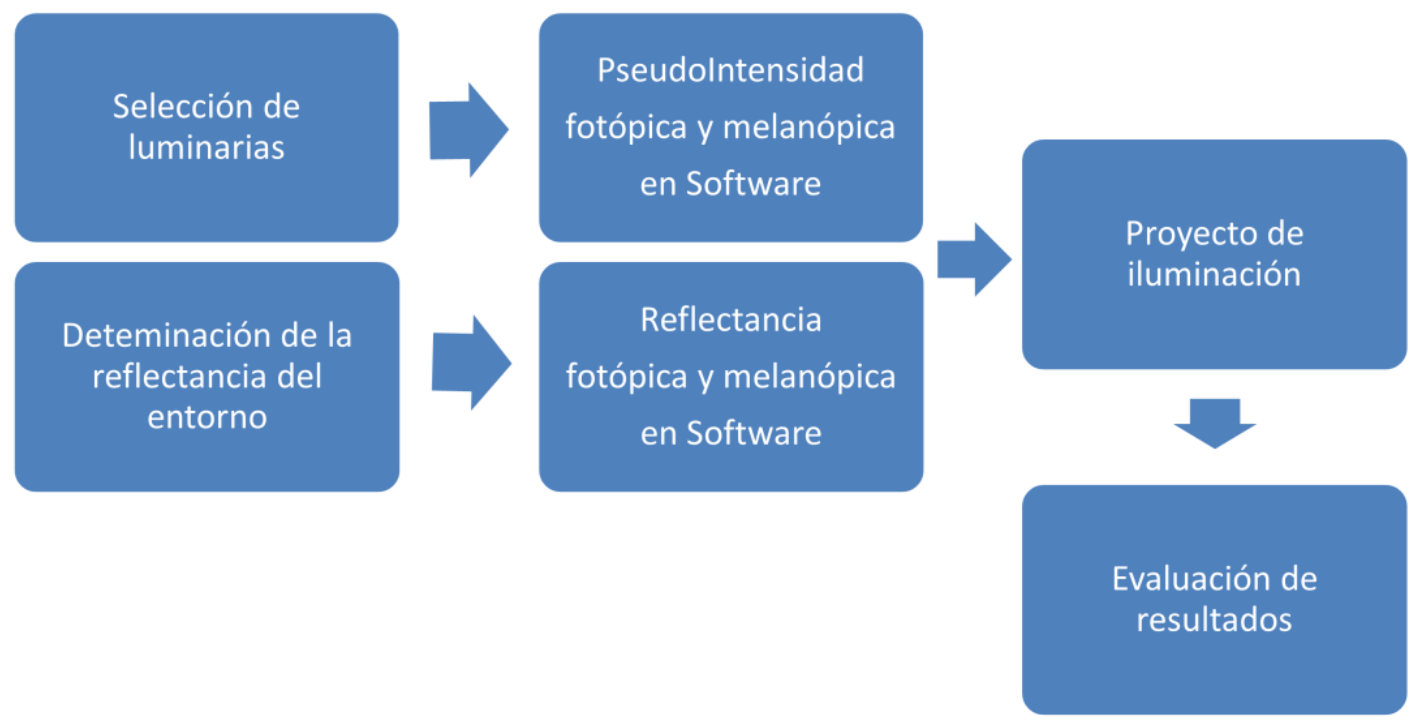

Fig.6. Diagrama de flujo con el método propuesto para realizar los cálculos fotópicos y melanópicos en los proyectos de iluminación.

\section{Discusión}

Son muchos e importantes los efectos que la luz y la iluminación tienen sobre los organismos vivos, y particularmente sobre los seres humanos, yendo más allá del sistema visual formador de imagen. En la retina, además de los conos y bastones, responsables de estas funciones visuales, están las células ipRGC presentes en toda la retina del ojo, más sensibles a la radiación de longitudes de onda de la zona de los azules, con el pico de máxima sensibilidad en $480 \mathrm{~nm}$, responsables de la entrada por el ojo de la luz 
necesaria para, a través de una ruta no formadora de imagen, regular el reloj biológico. Si bien no tenemos suficiente conocimiento de los efectos fotobiológicos y no visuales de la luz, se debe analizar la cantidad de luz que puede llegar a la córnea [25] y, lo que es más importante, a la retina. La diferente distribución retiniana de las ipRGC y su población, dependiente de la edad, indican que la exposición nasal es más efectiva que la exposición temporal, y la exposición inferior a la luz parece inducir una respuesta mayor que la exposición superior [26]. La direccionalidad es un factor de luz importante para la salud humana a considerar en el diseño de iluminación [33]. En los proyectos de iluminación de edificios, y dependiendo de la magnitud de la influencia que se esté buscando, este factor se debe tener en cuenta porque la elección de la posición correcta de las lámparas de lectura, las luminarias y las aberturas de luz diurna puede influir claramente en la salud y el bienestar de los individuos. El color del entorno, los tipos de muebles, o incluso, la reflectancia del formato de lectura (papel, libro electrónico, pantallas, ...), deben considerarse como fuentes secundarias de contribuciones melanópicas para el diseño de iluminación en el entorno de cualquier edificio. Esto puede afectar a la actividad cotidiana, así como a muchas funciones reguladas por los sistemas neurovegetativo y somático, y tienen incidencia en actividades y bienestar a corto, medio y largo plazo [19,27-29].

Especificar y medir la contribución melanópica de la luz en un determinado ambiente se ha convertido en un factor a tener en cuenta [30]. El gran incremento de los tiempos de medida del espectro, tanto para caracterizar las luminarias, como para conocer la luz que llega a la pupila del ojo situado en una estancia iluminada, obligan a plantear un detector con un filtro melanópico, similar al fotópico que se utilice para la medida de la intensidad lumínica, pero con otra distribución, capaz de dar directamente la intensidad melanópica. Dado que la distribución espacial de intensidades de muchas luminarias, que lleven incorporados elementos dispersivos como lentes o prismas [31,32], puede depender del ángulo y de la longitud de onda requiere que esta información provenga directamente del fabricante. Esta problemática acercaría más la posibilidad de que los fabricantes de luminarias pudiesen caracterizar sus luminarias con los dos tipos de ficheros, y de forma sencilla con pocas modificaciones, especificar ambas contribuciones: la fotópica y la melanópica. Es nuestro objetivo que, a partir de ese conocimiento, utilizando las luminarias, los sistemas de control apropiados, y el entorno adecuado [19], en los proyectos de iluminación de interiores se puedan introducir las variaciones temporales, diarias y estacionales, a las que los seres humanos han estado sometidos durante años y que según se está demostrando tanta influencia tienen en el comportamiento, bienestar y salud. Por una parte, la orientación en el tiempo, modificando el periodo intrínseco (horario) de nuestro reloj biológico y, por otra, influyendo en la producción de diversas hormonas con origen en diferentes glándulas, como el cortisol, la melatonina y la serotonina (esta última es más bien neurohormona o neurotransmisor), que parecen jugar un importante papel en el control del humor o estado de ánimo, en la regulación de patrón de sueño, en la temperatura corporal, en el sistema metabólico y endocrino, o reproductor [34-36].

\section{Conclusiones}

En conclusión, hemos desarrollado un método sencillo para incluir la contribución melanópica en el diseño de la iluminación, haciéndolo más intuitivo para investigadores, arquitectos y diseñadores de sistemas de iluminación de interiores, a partir de determinadas especificaciones en entornos arquitectónicos enfocados a valorar los potenciales efectos circadianos y melanópicos. Proponemos el uso de los valores de reflectancia del entorno y del SPD de las luminarias, además de curvas polares melanópicas, en el uso de software estándar como puede ser DIALux. Son muy numerosos los programas de cálculo de iluminación disponibles en el mercado y la metodología aquí propuesta podría ser extensible a cualquiera de ellos modificando, en cada caso, los distintos archivos fotométricos IES, CIB o ULD. Hemos comprobado nuestro método con DIALux comparándolo con el Estándar de Construcción WELL (basado en las descripciones de Lucas et al. [18]) como un caso de estudio para demostrar la validez de nuestra propuesta.

\section{Agradecimientos}

Partes de este trabajo fueron presentadas en el congreso LED professional Symposium 2017 en Melanopic Photometry vs. Melanopic Lux - The WELL Standard as a Case Study, 2017. 